\title{
Jahrestagung 1970
}

In den Tagen vom 7. bis 10 . Oktober 1970 fand die Jahrestagung der Vereinigung der Deutschen Staatsrechtslehrer in Speyer statt. Zu Beginn der Tagung begrüßte der Vorsitzende der Vereinigung, Herr Schneider (Heidelberg), die anwesenden Mitglieder, als Gäste weitere Kollegen, die an der Hochschule für Verwaltungswissenschaften in Speyer tätig sind, und die Vertreter der Fachzeitschriften. Er dankte dem Rektor der Hochschule für Verwaltungswissenschaften, Herrn Knöpfle, den weiteren Mitgliedern ihres Lehrkörpers und dem Personal der Hochschule für die überaus sorgfältige und aufmerksame Vorbereitung und die großzügige Ausrichtung der Tagung. Die Tagung war durch keine Störung getrübt. Sie fand in den modernen Räumen der Hochschule einen recht förderlichen technischen und architektonischen Rahmen.

Die Tagung bot zahlreiche Gelegenheiten zu gesellschaftlicher Begegnung. Die Hochschule für Verwaltungswissenschaften lud die Teilnehmer und ihre Damen zu einem Begrüßungsessen in die Räume der Hochschule. Der Ministerpräsident des Landes Rheinland-Pfalz begrüßte und bewirtete die Teilnehmer und ihre Damen in Frankenthal. Namens der Stadt Speyer wurden sie vom Oberbürgermeister im Rathaus nicht weniger gastlich empfangen. Durch den Speyerer Dom führte der Domprobst. Am Samstag, dem 10. Oktober, bereisten die Teilnehmer und ihre Damen noch das schöne Pfälzer Land. Bei all dem bot sich für Gastgeber und Gäste immer wieder Gelegenheit, Erfahrungen, Sorgen, Wohlwollen und Hoffnungen auszutauschen.

In der Mitgliederversammlung wurde der seit der letzten Jahrestagung verstorbenen Mitglieder der Vereinigung, Fritz Werner, Erich Kordt, Helfried Pfeifer, Wilhelm Merk, Rudolf Petz, Adolf Julius Merkl und Franz Jerusalem gedacht. Zahlreiche neue Mitglieder wurden der Vereinigung vorgestellt, darunter ihr erstes weibliches Mitglied, Frau Staff (Frankfurt).

Die nachstehend abgedruckten Referate wurden am 8. und 9. Oktober in der Hochschule für Verwaltungswissenschaften gehalten. An sie schlossen sich jeweils die Aussprachen an. 
